\title{
Estimating the thermodynamic properties of phosphate minerals at high and low temperature from the sum of constituent units
}

\section{Estimación de las propiedades termodinámicas de fosfatos minerales a alta y baja temperatura por suma de sus unidades constituyentes}

\author{
A. La Iglesia ${ }^{1}$
}

\section{RESUMEN}

\begin{abstract}
Usando el modelo de unidades poliédricas de Hazen, y empleando un método de ajuste por mínimos cuadrados, se ha calculado la contribución de diecinueve unidades constituyentes a la energía libre y de otras quince unidades a la entalpía de los fosfatos minerales en condiciones termodinámicas normales (298 ${ }^{\circ} \mathrm{K}$ de temperatura y 1 bar de presión) y a altas temperaturas $\left(400-1.000{ }^{\circ} \mathrm{K}\right)$.

Con los valores de $g_{i}$ y $h_{i}$ calculados por este método se puede estimar las propiedades termodinámicas de cualquier fosfato mineral con mayor precisión que la conseguida hasta ahora por otros métodos.
\end{abstract}

Palabras clave: Energía libre de Gibbs, Entalpía, Fosfatos minerales, Propiedades termodinámicas.

\section{ABSTRACT}

Using the polyhedral units model of Hazen and employing a method of least-squares, the contribution of nineteen constituent units to the free energy and fifteen units to the enthalpy, at $298 \mathrm{~K}$ and 1 bar of pressure, have been calculated for mineral phosphates. The contribution of these constituent units to the free energy at higher temperatures has also been calculated.

From these data we can estimate the thermodynamic properties of phosphates by summing the contribution of the distinct units, with more accuracy than the methods published up until now.

Key words: Gibbs Free Energy, Enthalpy, Phosphate minerals, Thermodynamic properties.

\section{Introduction}

The estimation of the thermodynamic properties of silicate minerals has been considered by many investigators. Wilcox \& Bromley (1963), Slaughter (1966), Karpov \& Kashik (1968), Tardy \& Garrels (1974), Nriagu (1975), Chen (1975), Robinson \& Haas (1983), Sposito (1986) and La Iglesia \& Aznar (1986, 1990) have published methods based on the hypothesis that the free energy or enthalpy of formation is related to the sum of free energies of formation of their constituent oxide or hydroxide components. This summation hypothesis has a firm theoretical backing in that the macroscopic mineral thermodynamic properties are the cumulative result of the lattice vibrational energies of the various elements in the lattice, Grimwall (2001).

Following the polyhedral units model of Hazen (1985), Chermak \& Rimstidt (1989, 1990), determined by multiple linear regression the Gibbs free energy of formation $\left(\mathrm{g}_{\mathrm{i}}\right)$ and enthalpy of formation $\left(\mathrm{h}_{\mathrm{i}}\right)$ of polyhedral units in silicate minerals:

$$
\Delta \mathrm{G}_{\mathrm{f}}^{\circ}=\Sigma \mathrm{n}_{\mathrm{i}} \mathrm{g}_{\mathrm{i}}
$$

and

$$
\Delta \mathrm{H}_{\mathrm{f}}^{\circ}=\Sigma \mathrm{n}_{\mathrm{i}} \mathrm{h}_{\mathrm{i}}
$$

\footnotetext{
1 Instituto de Geología Económica. CSIC. Facultad de Ciencias Geológicas. Universidad Complutense. 28040 Madrid. Email: iglesia@geo.ucm.es
} 
where $n_{i}$ is the number of moles of ith component per formula unit. With this method $\Delta \mathrm{G}_{\mathrm{f}}^{\circ}$ or $\Delta \mathrm{H}_{\mathrm{f}}^{\circ}$ of many silicates can be calculated with an uncertainty of less than $1 \%$. Using this approach, La Iglesia \& Felix (1994) calculated values of $g_{i}$ and hi for carbonates to create a model that predicts their $\Delta \mathrm{G}_{\mathrm{f}}^{\circ}$ and $\Delta \mathrm{H}_{\mathrm{f}}^{\circ}$ with an uncertainty of less than $0.5 \%$.

Tardy \& Garrels $(1976,1977)$ published a method of estimation of the thermodynamic properties of minerals based on the fact that the free energy and enthalpy of formation within a family of compounds from their constituent oxides are linearly dependent on a parameter called $\Delta \mathrm{O}_{\mathrm{M}}{ }^{2-}$, which is a function of the electronegativity of the constituent cations $\left(\mathrm{M}^{2+}\right)$. This correlation provides a means of estimating values for unmeasured minerals. So, Tardy \& Gartner (1977) and Tardy \& Vieillard (1977) have obtained empirical equations that estimate the Gibbs free energy of formation and enthalpy of formation of carbonates, nitrates, sulfates and phosphates with a deviation less than $3 \%$ with respect to the experimental values. For the phosphates, these authors obtain the following equations:

$$
\begin{gathered}
\left.\Delta \mathrm{G}_{\mathrm{f}}^{\circ} \text { (phosphate }\right)=\Delta \mathrm{G}_{\mathrm{f}}^{\circ} \text { (oxides) }- \\
1.40 \mathrm{n}_{1} \mathrm{n}_{2}\left(\Delta_{\mathrm{G}} \mathrm{O}_{\mathrm{M}}^{2-}+76.9\right) /\left(\mathrm{n}_{1}+\mathrm{n}_{2}\right)(\mathrm{kcal} / \mathrm{mol})
\end{gathered}
$$

and

$$
\begin{gathered}
\Delta \mathrm{H}_{\mathrm{f}}^{\circ}(\text { phosphate })=\Delta \mathrm{H}_{\mathrm{f}}^{\circ}(\text { oxides })- \\
1.41 \mathrm{n}_{\mathrm{l}} \mathrm{n}_{2}\left(\Delta_{\mathrm{H}} \mathrm{O}_{\mathrm{M}}^{2-}+87.3\right) /\left(\mathrm{n}_{\mathrm{l}}+\mathrm{n}_{2}\right)(\mathrm{kcal} / \mathrm{mol})
\end{gathered}
$$

in which $\mathrm{n}_{1}$ and $\mathrm{n}_{2}$ are in a given compound, the number of oxygens required to balance the cation and phosphorous, respectively.

Nriagu (1976), employing the hypothesis of sum of the free energy of constituents, proposed the following equation, which permit to estimate the $\Delta \mathrm{G}_{\mathrm{f}}^{\circ}$ of complex phosphates and hydroxyphosphates:

$$
\begin{gathered}
\Delta \mathrm{G}^{\circ}{ }_{\mathrm{f}}(\text { hydroxyphosphate })= \\
\Sigma \Delta \mathrm{G}^{\circ}{ }_{\mathrm{f}(\mathrm{ri})}+\mathrm{n} \Delta \mathrm{G}^{\circ}{ }_{\mathrm{f}} \mathrm{H}_{2} \mathrm{O}-\mathrm{Q}
\end{gathered}
$$

where $\Delta \mathrm{G}_{\mathrm{f}(\mathrm{ri})}^{\circ}$ is the Gibbs free energy of formation of the ith reactant hydroxide or phosphate component within the hydroxyphosphate matrix and Q is a correction factor. According to Chen (1975), $\mathrm{Q}=\mathrm{RT} \sum\left(\mathrm{n}_{\mathrm{i}} \operatorname{lnn}_{\mathrm{i}}\right)$ where $\mathrm{n}_{\mathrm{i}}$ is the reaction coefficient for the ith hydroxide or phosphate. Using equation (5), values of $\Delta \mathrm{G}_{\mathrm{f}(\text { hydroxyphosphate) }}^{\circ}$ with deviations less than $3 \%$ with regard to experimental values, can be obtained.
In this paper, employing a method of leastsquares, the contribution of 19 constituent units to the Gibbs free energy and 15 units to the enthalpy of phosphates has been calculated. From these data we can estimate the thermodynamic properties of other phosphates with more accuracy than the methods published up until now.

\section{Calculation of the Gibbs free energies. enthalpies and entropies of phosphate units at $298^{\circ} \mathrm{K}$}

To evaluate the contribution of the units to the Gibbs free energy and enthalpy of phosphate minerals 41 crystalline phases (table 1) were selected. A system of 31 linear equations was established to calculate $\mathrm{g}_{\mathrm{i}}$ and 23 linear equations were used to calculate $\mathrm{h}_{\mathrm{i}}$. To solve both systems we used the program MAPLE $V$ release 2 . The $g_{i}$ and $h_{i}$ coefficients were determined using a least-squares method.

The values of $g_{i}$ and $h_{i}$ obtained for each of the polyhedral units, together with their uncertainty ranges, are presented in table 2 . Considering the easy substitution of $\mathrm{O}$ ligand by $\mathrm{OH}, \mathrm{F}$ or $\mathrm{Cl}$ in these compounds, average values of $\mathrm{g}_{2} \mathrm{O}_{(\mathrm{OH})}, \mathrm{g} \mathrm{F}$, $\mathrm{g} \mathrm{Cl}, \mathrm{h} \mathrm{H}_{2} \mathrm{O}_{(\mathrm{OH})}, \mathrm{h} \mathrm{F}$ and $\mathrm{h} \mathrm{Cl}$ have been also calculated. These values represent the difference between the energy of polyhedral units $\mathrm{M}-\mathrm{O}_{\mathrm{n}}$ and $M-\mathrm{O}_{\mathrm{n}-1}(\mathrm{OH})$ or $\mathrm{M}-\mathrm{O}_{\mathrm{n}-1} \mathrm{~F}$. Therefore, the procedure to calculate $\mathrm{g} \mathrm{Ca}(\mathrm{OH})_{2}$ for example, would be: $\mathrm{g}$ $\mathrm{Ca}(\mathrm{OH})_{2}=\mathrm{g} \mathrm{CaO}+\mathrm{g} \mathrm{H}_{2} \mathrm{O}_{(\mathrm{OH})}$. From the values of table 2 , the $\Delta \mathrm{G}_{\mathrm{f}}^{\circ}$ or $\Delta \mathrm{H}_{\mathrm{f}}^{\circ}$ of any phosphate can be easily calculated using the equations (1) and (2). Thus, for example, to calculate the standard thermodynamic properties of ammonium taranakite, $\left(\mathrm{NH}_{4}\right)_{3} \mathrm{Al}_{5}\left(\mathrm{PO}_{4}\right)_{8} \mathrm{H}_{6} 18 \mathrm{H}_{2} 0$ :

$$
\begin{gathered}
\Delta \mathrm{G}_{\mathrm{f}}^{\circ}\left(\mathrm{NH}_{4}\right)_{3} \mathrm{Al}_{5}\left(\mathrm{PO}_{4}\right)_{8} \mathrm{H}_{6} 18 \mathrm{H}_{2} \mathrm{O}=3 / 2 \mathrm{~g}\left(\mathrm{NH}_{4}\right)_{2} \mathrm{O}+ \\
5 / 2 \mathrm{~g} \mathrm{Al}_{2} \mathrm{O}_{3}+4 \mathrm{~g} \mathrm{P}_{2} \mathrm{O}_{5}+3 \mathrm{~g} \mathrm{H}_{2} \mathrm{O}_{(\mathrm{H})}+18 \mathrm{~g} \mathrm{H}_{2} \mathrm{O}_{\text {(cryst. }}= \\
-16,106.33 \pm 41.60 \mathrm{~kJ} \mathrm{~mol}^{-1} .
\end{gathered}
$$

$\Delta \mathrm{H}_{\mathrm{f}}^{\circ}\left(\mathrm{NH}_{4}\right)_{3} \mathrm{Al}_{5}\left(\mathrm{PO}_{4}\right)_{8} \mathrm{H}_{6} 18 \mathrm{H}_{2} \mathrm{O}=3 / 2 \mathrm{~h}\left(\mathrm{NH}_{4}\right)_{2} \mathrm{O}+$ $5 / 2 \mathrm{~h} \mathrm{Al}_{2} \mathrm{O}_{3}+4 \mathrm{~h} \mathrm{P}_{2} \mathrm{O}_{5}+3 \mathrm{~h} \mathrm{H}_{2} \mathrm{O}_{(\mathrm{H})}+18 \mathrm{~h} \mathrm{H}_{2} \mathrm{O}_{\text {(cryst. }}=$ $-18,484.51 \pm 38.45 \mathrm{~kJ} \mathrm{~mol}^{-1}$.

These results are in perfect agreement with the values of $-16,129.15 \mathrm{~kJ} \mathrm{~mol}^{-1}$ and $-18,532.60 \mathrm{~kJ}$ $\mathrm{mol}^{-1}$ given by Vieillard \& Tardy (1984). The deviations have been calculated using the equation:

$$
\sigma=\left[\Sigma\left(n_{\mathrm{i}} \sigma_{\mathrm{i}}\right)^{2}\right]^{1 / 2}
$$


Table 1.-Crystalline phases selected to calculate $g_{i}$ and $h_{i}$ values

\begin{tabular}{|c|c|c|c|}
\hline $\mathrm{Li}_{3} \mathrm{PO}_{4}$ (Lithiophosphatite) & $\mathrm{h}_{\mathrm{i}}$ & $\mathrm{Ca}_{10}\left(\mathrm{PO}_{4}\right)_{6} \mathrm{~F}_{2}$ (Fluorapatite) & $\mathrm{g}_{\mathrm{i}}+\mathrm{h}_{\mathrm{i}}$ \\
\hline $\mathrm{Li}_{4} \mathrm{P}_{2} \mathrm{O}_{7}$ & $\mathrm{~h}_{\mathrm{i}}$ & $\mathrm{Ca}_{10}\left(\mathrm{PO}_{4}\right)_{6} \mathrm{Cl}_{2}$ (Chorapatite) & $\mathrm{g}_{\mathrm{i}}+\mathrm{h}_{\mathrm{i}}$ \\
\hline $\mathrm{Na}_{4} \mathrm{P}_{2} \mathrm{O}_{7} \cdot 10 \mathrm{H}_{2} \mathrm{O}$ & $\mathrm{h}_{\mathrm{i}}$ & $\mathrm{CaIIPO}_{4} \cdot 2 \mathrm{H}_{2} \mathrm{O}$ (Brushite) & $\mathrm{g}_{\mathrm{i}}+\mathrm{h}_{\mathrm{i}}$ \\
\hline $\mathrm{Na}_{5} \mathrm{P}_{3} \mathrm{O}_{10}$ & $\mathrm{~g}_{\mathrm{i}}+\mathrm{h}_{\mathrm{i}}$ & $\mathrm{Ca}_{2} \mathrm{C}_{1} \mathrm{PO}_{4}$ & $\mathrm{~g}_{\mathrm{i}}$ \\
\hline $\mathrm{Na}_{2} \mathrm{HPO}_{4} \cdot 12 \mathrm{H}_{2} \mathrm{O}$ & $\mathrm{g}_{\mathrm{i}}+\mathrm{h}_{\mathrm{i}}$ & $\mathrm{Fe}_{3}\left(\mathrm{PO}_{4}\right)_{2} \cdot 8 \mathrm{H}_{2} \mathrm{O}$ (Vivianite) & $\mathrm{g}_{\mathrm{i}}+\mathrm{h}_{\mathrm{i}}$ \\
\hline $\mathrm{Na}_{2} \mathrm{H}_{2} \mathrm{P}_{2} \mathrm{O}_{7}$ & $\mathrm{~g}_{\mathrm{i}}$ & $\mathrm{Fe}_{3}\left(\mathrm{PO}_{4}\right)_{2}$ & $\mathrm{~g}_{\mathrm{i}}$ \\
\hline $\mathrm{Na}_{3} \mathrm{HP}_{2} \mathrm{O}_{7}$ & $\mathrm{~h}_{\mathrm{i}}$ & $\mathrm{Co}_{3}\left(\mathrm{PO}_{4}\right)_{2}$ & $\mathrm{~g}_{\mathrm{i}}$ \\
\hline $\mathrm{KMgPO}_{4} \cdot 6 \mathrm{H}_{2} \mathrm{O}$ & $\mathrm{h}_{\mathrm{i}}$ & $\mathrm{Co}\left(\mathrm{UO}_{2}\right)_{2}\left(\mathrm{PO}_{4}\right)_{2} \cdot 7 \mathrm{H}_{2} \mathrm{O}$ & $\mathrm{g}_{\mathrm{i}}$ \\
\hline $\mathrm{K}_{2} \mathrm{HPO}_{4}$ & $\mathrm{~h}_{\mathrm{i}}$ & $\mathrm{Ni}_{2} \mathrm{P}_{2} \mathrm{O}_{7}$ & $\mathrm{~g}_{\mathrm{i}}$ \\
\hline $\mathrm{KUO}_{2} \mathrm{PO}_{4} \cdot 3 \mathrm{H}_{2} \mathrm{O}$ & $\mathrm{g}_{\mathrm{i}}$ & $\mathrm{Ni}\left(\mathrm{UO}_{2}\right)_{2}\left(\mathrm{PO}_{4}\right)_{2} \cdot 7 \mathrm{H}_{2} \mathrm{O}$ & $\mathrm{g}_{\mathrm{i}}$ \\
\hline $\mathrm{KAI}_{2}\left(\mathrm{PO}_{4}\right)_{2} \mathrm{OH} \cdot 2 \mathrm{H}_{2} \mathrm{O}$ (Minyulite) & $\mathrm{g}_{\mathrm{i}}$ & $\mathrm{Zn}_{3}\left(\mathrm{PO}_{4}\right)_{2} \cdot 4 \mathrm{H}_{2} \mathrm{O}$ (Hopeite) & $\mathrm{g}_{\mathrm{i}}+\mathrm{h}_{\mathrm{i}}$ \\
\hline $\mathrm{NH}_{4} \mathrm{MgPO}_{4} \cdot 6 \mathrm{H}_{2} \mathrm{O}$ (Struvite) & $\mathrm{g}_{\mathrm{i}}$ & $\mathrm{Zn}_{3}\left(\mathrm{PO}_{4}\right)_{2}$ & $\mathrm{~g}_{\mathrm{i}}$ \\
\hline $\mathrm{NH}_{4} \mathrm{Al}_{2}\left(\mathrm{PO}_{4}\right)_{2} \mathrm{OH} \cdot 2 \mathrm{H}_{2} \mathrm{O}$ (Am. Minyulite) & $\mathrm{g}_{\mathrm{i}}$ & $\mathrm{Zn}_{2} \mathrm{P}_{2} \mathrm{O}_{7}$ & $\mathrm{~h}_{\mathrm{i}}$ \\
\hline $\mathrm{NH}_{4} \mathrm{UO}_{2} \mathrm{PO}_{4} \cdot 3 \mathrm{H}_{2} \mathrm{O}$ & $\mathrm{g}_{\mathrm{i}}$ & $\mathrm{Cu}_{3}\left(\mathrm{PO}_{4}\right)_{2}$ & $\mathrm{~g}_{\mathrm{i}}+\mathrm{h}_{\mathrm{i}}$ \\
\hline $\mathrm{NH}_{4} \mathrm{H}_{2} \mathrm{PO}_{4}$ & $\mathrm{~h}_{\mathrm{i}}$ & $\mathrm{Cu}_{2} \mathrm{P}_{2} \mathrm{O}_{7}$ & $\mathrm{~g}_{\mathrm{i}}$ \\
\hline$\left(\mathrm{NH}_{4}\right)_{2} \mathrm{HPO}_{4}$ & $h_{i}$ & $\mathrm{Cu}\left(\mathrm{H}_{2} \mathrm{PO}_{4}\right)_{2}$ & $\mathrm{~h}_{\mathrm{i}}$ \\
\hline $\mathrm{Mg}_{3}\left(\mathrm{PO}_{4}\right)_{2}$ (Farringtonite) & $\mathrm{g}_{\mathrm{i}}+\mathrm{h}_{\mathrm{i}}$ & $\mathrm{Pb}_{3}(\mathrm{PO})_{2}$ & $\mathrm{~g}_{\mathrm{i}}+\mathrm{h}_{\mathrm{i}}$ \\
\hline $\mathrm{Mg}_{4}\left(\mathrm{PO}_{4}\right)_{2}$ & $\mathrm{~g}_{\mathrm{i}}$ & $\mathrm{Pb}_{4}\left(\mathrm{PO}_{4}\right)_{3}$ & $\mathrm{~g}_{\mathrm{i}}$ \\
\hline $\mathrm{Mg}_{5}\left(\mathrm{PO}_{4}\right)_{3} \mathrm{~F}$ & $\mathrm{~g}_{\mathrm{i}}$ & $\mathrm{A}_{4}\left(\mathrm{PO}_{4}\right)_{3}(\mathrm{OH})_{3}$ (Trolleite) & $\mathrm{g}_{\mathrm{i}}+\mathrm{h}_{\mathrm{i}}$ \\
\hline $\mathrm{Mg}_{3}\left(\mathrm{PO}_{4}\right)_{2} \cdot 8 \mathrm{H}_{2} \mathrm{O}$ (Bobierrite) & $\mathrm{g}_{\mathrm{i}}$ & $\mathrm{A} 1 \mathrm{PO}_{4}$ (Berlinite) & $\mathrm{g}_{\mathrm{i}}+\mathrm{h}_{\mathrm{i}}$ \\
\hline $\mathrm{Ca}_{10}\left(\mathrm{PO}_{4}\right)_{6}(\mathrm{OH})_{2}$ (Hydroxyapatite) & $\mathrm{g}_{\mathrm{i}}+\mathrm{h}_{\mathrm{i}}$ & & \\
\hline
\end{tabular}

To evaluate the accuracy of method a new set of individual contributions for $g_{i}$ and $h_{i}$ using another 18 crystalline phases has been calculated and compared to results of table 2 . The averages of differences are $0.62 \%$ for $\mathrm{g}_{\mathrm{i}}$ and $0.43 \%$ for $\mathrm{h}_{\mathrm{i}}(\mathrm{N}=10$ values considered each).

The values of $g_{i}$ and $h_{i}$ of table 2 have also been checked for internal consistency versus values of Robie et al. (1979) of $\Delta \mathrm{G}_{\mathrm{f}}^{\circ}$ and $\Delta \mathrm{H}_{\mathrm{f}}^{\circ}$ free oxides obtaining the following relations:

$$
\begin{gathered}
\mathrm{g}_{\mathrm{i}}=1.042 \Delta \mathrm{G}_{\mathrm{f}}^{\circ}(\mathrm{oxides})-80.115 \mathrm{~kJ} \mathrm{~mol}^{-1} \\
(\mathrm{r}=0.965) \\
\mathrm{h}_{\mathrm{i}}=1.047 \Delta \mathrm{H}_{\mathrm{f}}^{\circ}(\text { oxides })-87.455 \mathrm{~kJ} \mathrm{~mol}^{-1} \\
(\mathrm{r}=0.967)
\end{gathered}
$$

equivalents to the equations (3) and (4).

In the same way in which the free energy or enthalpy of a compound has just been defined as the sum of the free energies or enthalpies of its constituent units, we can also define the entropy as: $\mathrm{S}=\Sigma \mathrm{n}_{\mathrm{i}} \mathrm{s}_{\mathrm{i}}$, where $\mathrm{s}_{\mathrm{i}}$ is the entropy of the constituent unit. Taking into account the thermodynamic equation: $\Delta \mathrm{G}=\Delta \mathrm{H}-\mathrm{T} \Delta \mathrm{S}$; it is easy to obtain the equation that relates the entropy of polyhedral units with free energy and enthalpy:

$\mathrm{s}_{\mathrm{i}}=\left(\mathrm{h}_{\mathrm{i}}-\mathrm{g}_{\mathrm{i}}\right) / \mathrm{T}$, but it is recommendable to derivate these partial entropies following Holland (1989). This leads to lower propagated uncertainty on the resulting estimates.
Table 2. $-g_{i}$ and $h_{i}$ values of each basic unit calculated by the method of least-squares $\left(\mathrm{kJ} \mathrm{mo1}{ }^{-1}\right)$

\begin{tabular}{lrrrr}
\hline \multicolumn{1}{c}{ basic unit } & \multicolumn{1}{c}{$\mathrm{g}_{\mathrm{i}}$} & error \pm & \multicolumn{1}{c}{$\mathrm{h}_{\mathrm{i}}$} & error \pm \\
\hline $\mathrm{P}_{2} \mathrm{O}_{5}$ & $-1,636.94$ & 8.44 & $-1,726.84$ & 7.22 \\
$\mathrm{Li}_{2} \mathrm{O}$ & - & - & -817.30 & 12.97 \\
$\mathrm{Na}_{2} \mathrm{O}$ & -665.22 & 4.54 & -719.50 & 3.40 \\
$\mathrm{~K}_{2} \mathrm{O}$ & -751.28 & 1.24 & -774.50 & 3.26 \\
$(\mathrm{NH} 4)_{2} \mathrm{O}$ & -359.62 & 6.12 & -541.28 & 9.72 \\
$\mathrm{H}_{2} \mathrm{O}(\mathrm{H})$ & -227.54 & 2.52 & -308.20 & 1.66 \\
$\mathrm{MgO}$ & -628.52 & 3.87 & -692.92 & 4.29 \\
$\mathrm{CaO}$ & -742.46 & 3.64 & -792.81 & 5.57 \\
$\mathrm{FeO}$ & -269.53 & 3.17 & -319.16 & - \\
$\mathrm{CoO}$ & -251.88 & 3.00 & - & - \\
$\mathrm{NiO}$ & -233.41 & 2.85 & - & - \\
$\mathrm{ZnO}$ & -338.13 & 2.71 & -392.62 & 4.06 \\
$\mathrm{CuO}$ & -135.98 & 3.26 & -171.82 & 3.42 \\
$\mathrm{PbO}$ & -242.41 & 3.36 & -289.49 & - \\
$\mathrm{UO} \mathrm{O}_{3}$ & $-1,183.37$ & 4.03 & - & - \\
$\mathrm{A} 1_{2} \mathrm{O}_{3}$ & $-1,613.88$ & 7.92 & $-1,780.92$ & 6.72 \\
$\mathrm{H}_{2} \mathrm{O}($ cryst. $)$ & -239.10 & 0.42 & -299.22 & 0.62 \\
$\mathrm{H}_{2} \mathrm{O}\left(\mathrm{OH}{ }^{-}\right)$ & -255.04 & 8.08 & -267.20 & 6.06 \\
$\mathrm{~F}=\mathrm{O}$ & -284.89 & 8.16 & -285.35 & 5.52 \\
$\mathrm{Cl}=\mathrm{O}$ & -66.40 & 7.18 & -52.39 & 16.87 \\
\hline
\end{tabular}

Due to the scarce number of data found in the bibliography for $\Delta \mathrm{G}_{\mathrm{f}}^{\circ}$ and $\Delta \mathrm{H}_{\mathrm{f}}^{\circ}$ (phosphate), the contribution of the cation coordination number to the Gibbs free energy values of polyhedral units (which is important in calcium, aluminium and lead phosphates where different types of coordination 
Table 3.-Temperature function $\mathrm{g}_{\mathrm{i} . \mathrm{T}}$ of the basic units $\left(\mathrm{kJ} \mathrm{mo1}{ }^{-1}\right)$

\begin{tabular}{lr}
\hline $\mathrm{P}_{2} \mathrm{O}_{5}$ & $-1,726.84+0.30 \mathrm{~T}$ \\
$\mathrm{Na}_{2} \mathrm{O}$ & $-719.80+0.18 \mathrm{~T}$ \\
$\mathrm{~K}_{2} \mathrm{O}$ & $-774.50+0.08 \mathrm{~T}$ \\
$\left(\mathrm{NH}_{4}\right)_{2} \mathrm{O}$ & $-541.28+0.61 \mathrm{~T}$ \\
$\mathrm{H}_{2} \mathrm{O}_{(\mathrm{H})}$ & $-308.20+0.27 \mathrm{~T}$ \\
$\mathrm{MgO}$ & $-692.92+0.21 \mathrm{~T}$ \\
$\mathrm{CaO}$ & $-792.81+0.17 \mathrm{~T}$ \\
$\mathrm{FeO}$ & $-319.16+0.17 \mathrm{~T}$ \\
$\mathrm{ZnO}$ & $-392.62+0.18 \mathrm{~T}$ \\
$\mathrm{CuO}$ & $-171.82+0.12 \mathrm{~T}$ \\
$\mathrm{PbO}$ & $-289.49+0.16 \mathrm{~T}$ \\
$\mathrm{~A} 1_{2} \mathrm{O}_{3}$ & $-1,780.92+0.55 \mathrm{~T}$ \\
$\mathrm{H}_{2} \mathrm{O}_{\text {(cryst. }}$ & $-299.22+0.20 \mathrm{~T}$ \\
$\mathrm{H}_{2} \mathrm{O}_{(\mathrm{OH})}$ & $-267.20+0.04 \mathrm{~T}$ \\
$\mathrm{~F} \equiv \mathrm{O}$ & $-285.35+0.002 \mathrm{~T}$ \\
$\mathrm{C} 1 \equiv \mathrm{O}$ & $-52.39-0.05 \mathrm{~T}$ \\
\hline
\end{tabular}

take place) could not be considered, as was done for the case of silicates and carbonates (Chermak \& Rimstidt, 1989 \& 1990 and La Iglesia \& Felix, 1994). For this reason, the precision of the values calculated for $g_{i}$ and $h_{i}$ phosphate, is lower than that of silicates and carbonates.

\section{Calculation of the free energies of polyhedral phosphate units at high temperature}

Chermak \& Rimstidt (1990) published a method that permits the estimation of free energy of any silicate at high temperature, based on the approximation $\Delta \mathrm{C} / \delta \mathrm{T}=0$, which gives for the free energy at the polyhedral units versus temperature:

$$
\mathrm{g}_{\mathrm{i} . \mathrm{T}}=\mathrm{h}_{\mathrm{i} .298}-\left[\mathrm{T}\left(\mathrm{h}_{\mathrm{i} .298}-\mathrm{g}_{\mathrm{i} .298}\right)\right] / 298
$$

This equation allows the calculation of $\Delta \mathrm{G}^{\circ}{ }_{\mathrm{f} \cdot \mathrm{T}}$ of any silicate, in the temperature range 298-650 $\mathrm{K}$, with an uncertainty of $0.25 \%$ (in relation to experimental value of Robie et al. (1979)). For temperatures higher than $650 \mathrm{~K}$ the estimated values of $\Delta \mathrm{G}^{\circ}{ }_{\mathrm{f} \cdot \mathrm{T}}$ can carry an uncertainty of $0.5 \%$. This method has been applied for the estimation $\Delta \mathrm{G}_{\mathrm{f} \cdot \mathrm{T}}^{\circ}$ in carbonates, in the temperature range 400 to $1.000 \mathrm{~K}$ with difference less $0.60 \%$ (La Iglesia $\&$ Felix, 1994). This is therefore an excellent method for estimating the $\Delta \mathrm{G}_{\mathrm{f} \cdot \mathrm{T}}^{\circ}$ of silicates and carbonates.

Using Equation 7, we have calculated the temperature function $g_{\text {i.T }}$ of the phosphate units, which appears in table 3 .

\section{Results}

Tables 4 and 5 compare the standard free energy and enthalpy values predicted in this paper with the bibliographic data; the differences are shown as percent of relative error (\% residual), $R=100\left(\Delta \mathrm{G}_{\text {cal }}\right.$ $\left.-\Delta \mathrm{G}_{\mathrm{bibl}}\right) / \Delta \mathrm{G}_{\mathrm{bibl}}$. When several experimental values of $\Delta \mathrm{G}_{\mathrm{f}}^{\circ}$ were found for one crystalline species we have calculated the residual error in relation to their average value $\left(\Delta \mathrm{G}_{\mathrm{bibl}}\right.$ or $\left.\Delta \mathrm{H}_{\mathrm{bibl}}\right)$. For $\Delta \mathrm{G}_{\mathrm{f}}^{\circ}$ values, the calculated average residual error is: $\mathrm{R}=0.029$, and standard deviation, $\Delta_{\mathrm{n}}=0.619(\mathrm{~N}=82$ values considered). For $\Delta \mathrm{H}^{\circ}, \mathrm{R}=-0.003$, and $\Delta_{\mathrm{n}}=0.525$ $(\mathrm{N}=58$ values considered). From the 82 values of $\Delta \mathrm{G}_{\mathrm{f}}^{\circ}$ estimated, 74 values have an error less than $1 \%, 7$ values are between 1 and $2 \%$ and only 1 value is more than $2 \%$. Similar distribution is found for $\Delta \mathrm{H}_{\mathrm{f}}^{\circ}$. All values are lower than $2 \%$, and only 3 are over $1 \%$. The preceding results confirm the goodness of the proposed method for the estimation of the thermodynamic properties of phosphates. Figures 1 and 2 present the frequency histogram of percent residual differences between bibliographic and calculated values for $\Delta \mathrm{G}^{\circ}$ and $\Delta \mathrm{H}^{\circ}$. In both cases, a Gaussian distribution is obtained.

The accuracy of the proposed method was tested by predicting the thermodynamic properties of the phosphates not used to calculate $\mathrm{g}_{\mathrm{i}}$ and $\mathrm{h}_{\mathrm{i}}$ contribution (in tables 4 and 5 the values of $\Delta \mathrm{G}_{\mathrm{f}}^{\circ}$ and $\Delta \mathrm{H}_{\mathrm{f}}^{\circ}$ used to calculate $g_{i}$ and $h_{i}$ have been marked with $*$ ). The average residual error and standard deviation calculated from the above data for $\Delta \mathrm{G}_{\mathrm{f}}^{\circ}$ and $\Delta \mathrm{H}_{\mathrm{f}}^{\circ}$ are: $\mathrm{R}=0.002$ and $\Delta_{\mathrm{n}}=0.697(\mathrm{~N}=51$ values considered) and $\mathrm{R}=0.075$ and $\Delta_{\mathrm{n}}=0.583$ $(\mathrm{N}=35$ values considered), respectively. The uncertainty ranges of these estimates data are larger that those obtained by La Iglesia \& Felix (1994) in the estimation of thermodynamic properties of carbonates. It was not possible to obtain better results for the following two reasons: 1) as indicated before, the contribution of the cation coordination to the Gibbs free energy values of the polyhedral units could not be considered as in the case of silicates and carbonates, and 2) the poorer agreement amoung the reported thermodynamic data for the phosphates. In this way, we have calculated the residual errors, in relation to the average values of $\Delta \mathrm{G}_{\mathrm{f}}^{\circ}$ and $\Delta \mathrm{H}_{\mathrm{f}}^{\circ}$ for some bibliographic data of fluorapatite and hydroxyapatite selected by Tacker \& Stormer (1989) obtaining the following values of standard deviation: $\sigma_{\mathrm{n}}=0.666$ (for $\Delta \mathrm{G}_{\mathrm{f}}^{\circ}, \mathrm{N}=17$ values considered) and $\sigma_{\mathrm{n}}=0.645$ (for $\Delta \mathrm{H}^{\circ}{ }_{\mathrm{f}}, \mathrm{N}=19$ 
Table 4.-Comparison of $\Delta \mathbf{G}_{\mathrm{f}}{ }^{0}$ (bibliography) versus $\Delta \mathbf{G}_{\mathrm{f}}{ }^{0}$ (calculated) for phosphates and residual error $\left(\mathrm{kJ} \mathrm{mol}^{-1}\right)$

\begin{tabular}{|c|c|c|c|c|}
\hline \multirow{2}{*}{$\frac{\text { Crystalline species }}{\mathrm{Na}_{3} \mathrm{PO}_{4}}$} & \multicolumn{2}{|l|}{$\Delta \mathrm{G}_{\mathrm{f}}^{0}($ bibli. $)$} & \multirow{2}{*}{$\frac{\left.\Delta \mathrm{G}_{\mathrm{f}}^{0} \text { (calc. }\right)}{-1,816.30 \pm 7.11}$} & \multirow{2}{*}{$\begin{array}{l}\mathrm{R} \% \\
1.54\end{array}$} \\
\hline & $-1,788.80$ & (1) & & \\
\hline $\mathrm{Na}_{4} \mathrm{P}_{2} \mathrm{O}_{7}$ & $-2,969.30$ & (1) & $-2,967.38 \pm 12.40$ & -0.07 \\
\hline $\mathrm{Na}_{5} \mathrm{P}_{3} \mathrm{O}_{10}$ & $-4,100.20 *$ & (1) & $-4,118.46 \pm 17.00$ & 0.45 \\
\hline $\mathrm{Na}_{5} \mathrm{P}_{3} \mathrm{O}_{10} \cdot 6 \mathrm{H}_{2} \mathrm{O}$ & $-5,540.10$ & (1) & $-5,553.06 \pm 17.19$ & 0.22 \\
\hline $\mathrm{NaH}_{2} \mathrm{PO}_{4}$ & $-1,386.10$ & (1) & $-1,378.62 \pm 5.41$ & -0.54 \\
\hline $\mathrm{Na}_{2} \mathrm{HPO}_{4}$ & $-1,608.20$ & (1) & $-1,597.46 \pm 6.21$ & -0.67 \\
\hline $\mathrm{Na}_{2} \mathrm{HPO}_{4} \cdot 2 \mathrm{H}_{2} \mathrm{O}$ & $-2,088.50$ & (1) & $-2,075.66 \pm 6.26$ & -0.62 \\
\hline $\mathrm{Na}_{2} \mathrm{HPO}_{4} \cdot 7 \mathrm{H}_{2} \mathrm{O}$ & $-3,279.80$ & (1) & $-3,271.16 \pm 6.98$ & -0.26 \\
\hline $\mathrm{Na}_{2} \mathrm{HPO}_{4} \cdot 12 \mathrm{H}_{2} \mathrm{O}$ & $-4,467.80 *$ & (1) & $-4,466.66 \pm 8.09$ & -0.03 \\
\hline $\mathrm{Na}_{2} \mathrm{H}_{2} \mathrm{P}_{2} \mathrm{O}_{7}$ & $-2,522.40$ & (1) & $-2,529.70 \pm 9.91$ & 0.29 \\
\hline \multirow[t]{2}{*}{$\mathrm{KH}_{2} \mathrm{PO}_{4}$} & $-1,415.85$ & (1) & $-1,421.65 \pm 4.95$ & 0.41 \\
\hline & $-1,418.30$ & (2) & & 0.17 \\
\hline $\mathrm{KUO}_{2} \mathrm{PO}_{4}$ & $-2,386.60$ & (2) & $-2,377.48 \pm 5.87$ & -0.38 \\
\hline $\mathrm{KUO}_{2} \mathrm{PO}_{4} \cdot 3 \mathrm{H}_{2} \mathrm{O}$ & $-3,098.20 *$ & (2) & $-3,094.78 \pm 6.00$ & -0.11 \\
\hline $\mathrm{K}_{3} \mathrm{Al}_{5}\left(\mathrm{PO}_{4}\right)_{8} \mathrm{H}_{6} \cdot 18 \mathrm{H}_{2} \mathrm{O}$ Pot. Taranakite & $-16,760.80$ & (2) & $-16,695.90 \pm 41.26$ & -0.39 \\
\hline $\mathrm{KA}_{2}\left(\mathrm{PO}_{4}\right)_{2} \mathrm{OH} \cdot 2 \mathrm{H}_{2} \mathrm{O}$ Minyulite & $-4,237.10 *$ & (2) & $-4,232.18 \pm 11.79$ & -0.12 \\
\hline $\mathrm{KMgPO}_{4} \cdot 6 \mathrm{H}_{2} \mathrm{O}$ & $-3,241.00$ & (2) & $-3,257.23 \pm 6.29$ & 0.50 \\
\hline \multirow[t]{2}{*}{$\mathrm{NH}_{4} \mathrm{H}_{2} \mathrm{PO}_{4}$} & $-1,210.38$ & (1) & $-1,225.26 \pm 5.79$ & 1.22 \\
\hline & $-1,210.56$ & (3) & & 1.21 \\
\hline $\mathrm{NH}_{4} \mathrm{UO}_{2} \mathrm{PO}_{4}$ & $-2,201.60$ & (2) & $-2,180.99 \pm 6.59$ & -0.94 \\
\hline $\mathrm{NH}_{4} \mathrm{UO}_{2} \mathrm{PO}_{4} \cdot 3 \mathrm{H}_{2} \mathrm{O}$ & $-2,907.60 *$ & (2) & $-2,898.29 \pm 6.71$ & -0.32 \\
\hline \multirow[t]{2}{*}{$\mathrm{NH}_{4} \mathrm{MgPO}_{4}$} & $-1,624.00$ & (2) & $-1,626.14 \pm 6.49$ & 0.13 \\
\hline & $-1,631.80$ & (4) & & -0.35 \\
\hline $\mathrm{NH}_{4} \mathrm{MgPO}_{4} \cdot 6 \mathrm{H}_{2} \mathrm{O}$ Struvite & $-3,051.10 *$ & (5) & $-3,060.74 \pm 6.96$ & 0.32 \\
\hline \multirow[t]{2}{*}{$\left(\mathrm{NH}_{4}\right)_{3} \mathrm{Al}_{5}\left(\mathrm{PO}_{4}\right) \mathrm{H}_{6} \cdot 18 \mathrm{H}_{2} \mathrm{O}$ Amm. Taranakite } & $-16,129.15$ & $(2)$ & $-16,106.33 \pm 41.60$ & -0.14 \\
\hline & $-16,164.80$ & (1) & & 0.36 \\
\hline $\mathrm{NH}_{4} \mathrm{AI}_{2}(\mathrm{PO} 4)_{2} \mathrm{OH} \cdot 2 \mathrm{H}_{2} \mathrm{O}$ Amm. Minyulite & $-4,044.70 *$ & (2) & $-4,035.69 \pm 12.66$ & -0.30 \\
\hline \multirow[t]{3}{*}{$\mathrm{Mg}_{3}\left(\mathrm{PO}_{4}\right)_{2}$ Farringtonite } & $-3,538.70 *$ & (1) & $-3,522.51 \pm 14.35$ & -0.46 \\
\hline & $-3,538.80 *$ & (5) & & -0.46 \\
\hline & $-3,548.40 *$ & (6) & & -0.73 \\
\hline $\mathrm{Mg}\left(\mathrm{H}_{2} \mathrm{PO}_{4}\right)_{2}$ & $-2,730.10$ & (7) & $-2,720.57 \pm 10.56$ & -0.35 \\
\hline $\mathrm{Mg}\left(\mathrm{H}_{2} \mathrm{PO}_{4}\right)_{2} \cdot 2 \mathrm{H}_{2} \mathrm{O}$ & $-3,200.30$ & (7) & $-3,198.76 \pm 10.60$ & -0.05 \\
\hline $\mathrm{Mg}\left(\mathrm{H}_{2} \mathrm{PO}_{4}\right)_{2} \cdot 4 \mathrm{H}_{2} \mathrm{O}$ & $-3,674.40$ & (7) & $-3,676.95 \pm 10.63$ & 0.07 \\
\hline $\mathrm{MgHPO}_{4}$ & $-1,577.30$ & (2) & $-1,560.77 \pm 5.80$ & -1.05 \\
\hline \multirow[t]{2}{*}{$\mathrm{MgHPO}_{4} \cdot 3 \mathrm{H}_{2} \mathrm{O}$ Newberyite } & $-2,288.90$ & (2) & $-2,278.06 \pm 6.01$ & -0.47 \\
\hline & $-2,297.00$ & (7) & & -0.82 \\
\hline \multirow{2}{*}{$\mathrm{Mg}_{3}\left(\mathrm{PO}_{4}\right)_{2} \cdot 8 \mathrm{H}_{2} \mathrm{O}$ Bobierrite } & $-5,443.40 *$ & (2) & $-5,435.28 \pm 14.74$ & -0.15 \\
\hline & $-5,450.50 *$ & (2) & & -0.28 \\
\hline $\mathrm{Mg}_{3}\left(\mathrm{PO}_{4}\right)_{2} \cdot 22 \mathrm{H}_{2} \mathrm{O}$ & $-8,751.80$ & (2) & $-8,782.61 \pm 17.07$ & 0.35 \\
\hline $\mathrm{Mg}_{5}\left(\mathrm{PO}_{4}\right)_{3} \mathrm{~F}$ & $-5,854.25 *$ & (7) & $-5,882.91 \pm 24.52$ & 0.49 \\
\hline $\mathrm{Mg}_{5}\left(\mathrm{PO}_{4}\right)_{3} \mathrm{OH}$ & $-5,758.00$ & (7) & $-5,725.55 \pm 23.47$ & -0.56 \\
\hline $\mathrm{Mg}_{4} \mathrm{O}\left(\mathrm{PO}_{4}\right)_{2}$ & $-4,172.70 *$ & (7) & $-4,151.03 \pm 17.63$ & -0.52 \\
\hline $\mathrm{Mg}_{2} \mathrm{PO}_{4} \mathrm{~F}$ Wagnerite & $-2,305.40$ & (7) & $-2,360.40 \pm 12.01$ & 2.39 \\
\hline \multirow[t]{5}{*}{$\mathrm{Ca}_{3}\left(\mathrm{PO}_{4}\right)_{2}$ low Whitlockite } & $-3,884.82$ & $(5)$ & $-3,864.33 \pm 13.80$ & -0.53 \\
\hline & $-3,884.70$ & (1) & & -0.52 \\
\hline & $-3,899.50$ & (8) & & -0.90 \\
\hline & $-3,889.40$ & (6) & & -0.64 \\
\hline & $-3,895.60$ & (9) & & -0.80 \\
\hline \multirow[t]{3}{*}{$\mathrm{CaHPO}_{4}$ Monetite } & $-1,679.90$ & (8) & $-1,674.71 \pm 5.71$ & -0.31 \\
\hline & $-1,680.00$ & (6) & & -0.31 \\
\hline & $-1,681.20$ & (1) & & -0.39 \\
\hline \multirow[t]{3}{*}{$\mathrm{CaHPO}_{4} \cdot 2 \mathrm{H}_{2} \mathrm{O}$ Brushite } & $-2,154.75 *$ & (2) & $-2,152.90 \pm 5.78$ & -0.09 \\
\hline & $-2,153.10 *$ & (8) & & -0.01 \\
\hline & $-2,153.80 *$ & (6) & & -0.04 \\
\hline \multirow[t]{2}{*}{$\mathrm{Ca}\left(\mathrm{H}_{2} \mathrm{PO}_{4}\right)_{2}$} & $-2,824.80$ & (5) & $-2,834.51 \pm 10.48$ & 0.34 \\
\hline & $2,812.10$ & (4) & & 0.79 \\
\hline
\end{tabular}

* Values selected to calculate $g_{i},(1)$ Wagman et al. (1982), (2) Vieillard \& Tardy (1984), (3) Wagman (1968), (4) Latimer (1952), (5) Parker et al. (1971), (6) Naumov et al. (1971), (7) Duff (1971a), (8) Rossini et al. (1952), (9) Duff (1972), (10) Robie et al. (1979), (11) Duff (1971b), (12) Tacker \& Stormer (1989), (13) Al-Borno \& Tomson (1994), (14) Woods \& Garrels (1987). 
Table 4.-Comparison of $\Delta \mathbf{G}_{\mathrm{f}}{ }^{0}$ (bibliography) versus $\Delta \mathbf{G}_{\mathrm{f}}{ }^{0}$ (calculated) for phosphates and residual error $\left(\mathrm{kJ} \mathrm{mol}^{-1}\right)$ (continuation)

\begin{tabular}{|c|c|c|c|}
\hline \multirow{2}{*}{$\frac{\text { Crystalline species }}{\mathrm{Ca}\left(\mathrm{H}_{2} \mathrm{PO}_{4}\right) 2 \mathrm{H}_{2} \mathrm{O}}$} & $\Delta \mathrm{G}_{\mathrm{f}}^{0}($ bibli.) & \multirow{2}{*}{$\frac{\left.\Delta \mathrm{G}_{\mathrm{f}}{ }^{0} \text { (calc. }\right)}{-3,073.60 \pm 10.49}$} & \multirow{2}{*}{ R\% } \\
\hline & $\begin{array}{l}-3,058.40 \\
-3058.18\end{array}$ & & \\
\hline $\mathrm{Ca}_{2} \mathrm{P}_{2} \mathrm{O}_{7}$ & $-3,132.00$ & $-3,121.87 \pm 11.15$ & -0.32 \\
\hline $\mathrm{Ca}_{4} \mathrm{O}\left(\mathrm{PO}_{4}\right)_{2}$ Hilgenstockite & $-4,588.00$ & $-4,606.79 \pm 16.83$ & 0.41 \\
\hline $\mathrm{Ca}_{2} \mathrm{PO}_{4} \mathrm{Cl}$ & $-2,371.70 *$ & $-2,369.80 \pm 11.06$ & -0.08 \\
\hline \multirow[t]{2}{*}{$\mathrm{Ca}_{10} \mathrm{O}\left(\mathrm{PO}_{4}\right)_{6}$ Oxiapatite } & $-12,272.10$ & $-12,335.45 \pm 44.34$ & 0.52 \\
\hline & $-12,307.80$ & & 0.22 \\
\hline \multirow[t]{2}{*}{$\mathrm{Ca}_{8} \mathrm{H}_{2}\left(\mathrm{PO}_{4}\right)_{5} \cdot 5 \mathrm{H}_{2} \mathrm{O}$} & $-12,263.30$ & $-12,273.56 \pm 38.73$ & 0.08 \\
\hline & $-12,261.20$ & & 0.10 \\
\hline $\mathrm{Ca}\left(\mathrm{UO}_{2}\right)_{2}\left(\mathrm{PO}_{4}\right)_{2} \cdot 10 \mathrm{H}_{2} \mathrm{O}$ Autunite & $-7,147.00$ & $-7,137.14 \pm 12.93$ & -0.14 \\
\hline $\mathrm{CaAlH}\left(\mathrm{PO}_{4}\right)_{2} \cdot 6 \mathrm{H}_{2} \mathrm{O}$ & $-4,722.20$ & $-4,734.71 \pm 10.40$ & 0.27 \\
\hline \multirow[t]{8}{*}{$\mathrm{Ca}_{10}\left(\mathrm{PO}_{4}\right)_{6} \mathrm{~F}_{2}$ Fluorapatite } & $-12,983.00 * \quad(2)$ & $-12,905.23 \pm 47.25$ & -0.60 \\
\hline & $-12,911.56 *(10)$ & & -0.05 \\
\hline & $-12,885.92 *(12)$ & & 0.15 \\
\hline & $-12,920.04 *(12)$ & & -0.11 \\
\hline & $-12,922.54 *(12)$ & & -0.13 \\
\hline & $-12,941.80 *(12)$ & & -0.28 \\
\hline & $-12,949.32 *(12)$ & & -0.34 \\
\hline & $-13,062.30 *(12)$ & & -1.20 \\
\hline \multirow[t]{8}{*}{$\mathrm{Ca}_{10}\left(\mathrm{PO}_{4}\right)_{6}(\mathrm{OH})_{2}$ Hydroxyapatite } & $-12,572.19 *(10)$ & $-12,590.50 \pm 45.07$ & 0.15 \\
\hline & $-12,677.00 *$ & & -0.68 \\
\hline & $-12,734.38 *(12)$ & & -1.12 \\
\hline & $-12,752.17 *(12)$ & & -1.26 \\
\hline & $-12,672.67 *(12)$ & & -0.64 \\
\hline & $-12,503.47 *(12)$ & & 0.70 \\
\hline & $-12,514.37 *(12)$ & & 0.61 \\
\hline & $-12,648.81 *(12)$ & & -0.46 \\
\hline \multirow[t]{4}{*}{$\mathrm{Ca}_{10}\left(\mathrm{PO}_{4}\right)_{6} \mathrm{Cl}_{2}$ Chlorapatite } & $-12,514.00 *(2)$ & $-12,468.26 \pm 43.05$ & -0.37 \\
\hline & $-12,403.00 *(12)$ & & 0.53 \\
\hline & $-12,447.50 *(12)$ & & 0.17 \\
\hline & $-12,403.07 *(12)$ & & 0.53 \\
\hline \multirow[t]{2}{*}{$\mathrm{Fe}_{3}\left(\mathrm{PO}_{4}\right)_{2} \cdot 8 \mathrm{H}_{2} \mathrm{O}$ Vivianite } & $-4,377.20 *(2)$ & $-4,358.32 \pm 13.15$ & -0.43 \\
\hline & $-4,340.94 *(13)$ & & 0.40 \\
\hline $\mathrm{Fe}_{3}\left(\mathrm{PO}_{4}\right)_{2}$ & $-2,444.80 *(2)$ & $-2,445.55 \pm 12.72$ & 0.03 \\
\hline $\mathrm{CoHPO}_{4}$ & $-1,182.00$ & $-1,184.12 \pm 5.33$ & 0.18 \\
\hline \multirow[t]{2}{*}{$\mathrm{Co}_{3}\left(\mathrm{PO}_{4}\right)_{2}$} & $-2,399.10 *$ & $-2,392.58 \pm 12.34$ & -0.27 \\
\hline & $-2,398.60 *$ & & -0.25 \\
\hline $\mathrm{Co}\left(\mathrm{UO}_{2}\right)_{2}\left(\mathrm{PO}_{4}\right)_{2} \cdot 7 \mathrm{H}_{2} \mathrm{O}$ & $-5,937.40 *$ & $-5,929.26 \pm 12.40$ & 0.05 \\
\hline \multirow[t]{2}{*}{$\mathrm{Ni}_{3}\left(\mathrm{PO}_{4}\right)_{2}$} & $-2,347.30$ & $-2,337.17 \pm 12.01$ & -0.43 \\
\hline & $-2,353.00$ & & -0.67 \\
\hline $\mathrm{Ni}_{2} \mathrm{P}_{2} \mathrm{O}_{7}$ & $-2,083.10 *$ & $-2,103.76 \pm 10.18$ & 0.99 \\
\hline $\mathrm{Ni}\left(\mathrm{UO}_{2}\right)_{2}\left(\mathrm{PO}_{4}\right)_{2} \cdot 7 \mathrm{H}_{2} \mathrm{O}$ & $-5,926.30 *$ & $-5,910.79 \pm 12.37$ & -0.26 \\
\hline $\mathrm{Zn}_{3}\left(\mathrm{PO}_{4}\right)_{2}$ & $-2,633.40$ & $-2,651.33 \pm 11.72$ & 0.68 \\
\hline $\mathrm{Zn}_{3}\left(\mathrm{PO}_{4}\right)_{2} \cdot \mathrm{H}_{2} \mathrm{O}$ & $-2,890.90$ & $-2,890.43 \pm 11.73$ & -0.01 \\
\hline $\mathrm{Zn}_{3}(\mathrm{PO} 4)_{2} \cdot 2 \mathrm{H}_{2} \mathrm{O}$ & $-3,143.30$ & $-3,129.52 \pm 11.75$ & -0.44 \\
\hline \multirow[t]{2}{*}{$\mathrm{Zn}_{3}(\mathrm{PO} 4)_{2} \cdot 4 \mathrm{H}_{2} \mathrm{O}$ Hopeite } & $-3,616.40 *$ & $-3,607.71 \pm 11.84$ & -0.24 \\
\hline & $-3,606.30 * \quad(2)$ & & 0.04 \\
\hline \multirow[t]{3}{*}{$\mathrm{Cu}_{3}\left(\mathrm{PO}_{4}\right)_{2}$} & $-2,051.60 *(14)$ & $-2,044.94 \pm 12.92$ & -0.32 \\
\hline & $-2,051.30 *$ & & -0.31 \\
\hline & $-2,066.60 *(14)$ & & -1.04 \\
\hline $\mathrm{Cu}_{2} \mathrm{P}_{2} \mathrm{O}_{7}$ & $-1,874.30 *$ & $-1,908.94 \pm 10.67$ & 1.84 \\
\hline $\mathrm{Cu}_{3}\left(\mathrm{PO}_{4}\right)_{2} \cdot 2 \mathrm{H}_{2} \mathrm{O}$ & $-2,564.80$ & $-2,523.14 \pm 12.95$ & -1.62 \\
\hline $\mathrm{Cu}_{3}\left(\mathrm{PO}_{4}\right)_{2} \cdot 3 \mathrm{H}_{2} \mathrm{O}$ & $-2,753.00 *$ & $-2,762.24 \pm 12.98$ & 0.33 \\
\hline $\mathrm{Cu}_{5}\left(\mathrm{PO}_{4}\right)_{3} \mathrm{OH}$ & $-3,261.80$ & $-3,262.93 \pm 21.03$ & 0.04 \\
\hline $\mathrm{Cu}\left(\mathrm{UO}_{2}\right)_{2}\left(\mathrm{PO}_{4}\right)_{2} \cdot 8 \mathrm{H}_{2} \mathrm{O}$ & $-6,071.20$ & $-6,052.48 \pm 12.28$ & -0.31 \\
\hline $\mathrm{Pb}_{3}\left(\mathrm{PO}_{4}\right)_{2}$ & $-2,364.00 *$ & $-2,364.17 \pm 13.15$ & 0.01 \\
\hline
\end{tabular}

* Values selected to calculate $g_{i}$ (1) Wagman et al. (1982), (2) Vieillard \& Tardy (1984), (3) Wagman (1968), (4) Latimer (1952), (5) Parker et al. (1971), (6) Naumov et al. (1971), (7) Duff (1971a), (8) Rossini et al. (1952), (9) Duff (1972), (10) Robie et al. (1979), (11) Duff (1971b), (12) Tacker \& Stormer (1989), (13) Al-Borno \& Tomson (1994), (14) Woods \& Garrels (1987). 
Table 4.-Comparison of $\Delta \mathbf{G}_{\mathrm{f}}{ }^{0}$ (bibliography) versus $\Delta \mathbf{G}_{\mathrm{f}}{ }^{0}$ (calculated) for phosphates and residual error $\left(\mathrm{kJ} \mathrm{mol}^{-1}\right)$ (continuation)

\begin{tabular}{|c|c|c|c|}
\hline Crystalline species & $\Delta \mathrm{G}_{\mathrm{f}}^{0}$ (bibli.) & $\Delta \mathrm{G}_{\mathrm{f}}^{0}($ calc. $)$ & $\mathrm{R} \%$ \\
\hline $\mathrm{Pb}_{4} 0\left(\mathrm{PO}_{4}\right)_{2}$ & $-2,582.80 * \quad(2)$ & $-2,606.58 \pm 15.87$ & 0.91 \\
\hline $\mathrm{Pb}_{5}\left(\mathrm{PO}_{4}\right)_{3} \mathrm{C} 1$ Pyromorphite & $-3,791.50$ & $-3,733.86 \pm 22.23$ & -1.52 \\
\hline $\mathrm{Pb}_{5}\left(\mathrm{PO}_{4}\right)_{3} \mathrm{OH}$ & $-3,774.10$ & $-3,794.98 \pm 21.42$ & 0.53 \\
\hline $\mathrm{PbHPO}_{4}$ & $-1,178.90$ & $-1,174.65 \pm 5.54$ & -0.36 \\
\hline$\left(\mathrm{UO}_{2}\right)_{3}\left(\mathrm{PO}_{4}\right)_{2}$ & $-5,160.00$ & $-5,187.05 \pm 14.74$ & 0.52 \\
\hline $\mathrm{H}_{2}\left(\mathrm{UO}_{2}\right)_{2}\left(\mathrm{PO}_{4}\right)_{2}$ & $-4,217.50$ & $-4,231.22 \pm 11.94$ & 0.33 \\
\hline $\mathrm{H}_{2}\left(\mathrm{UO}_{2}\right)_{2}\left(\mathrm{PO}_{4}\right)_{2} \cdot 10 \mathrm{H}_{2} \mathrm{O}$ & $-6,605.60$ & $-6,622.22 \pm 12.66$ & 0.25 \\
\hline $\mathrm{UO}_{2} \mathrm{HPO}_{4}$ & $-2,102.90$ & $-2,115.61 \pm 5.97$ & 0.60 \\
\hline $\mathrm{UO}_{2} \mathrm{HPO}_{4} \cdot 4 \mathrm{H}_{2} \mathrm{O}$ & $-3,051.30$ & $-3,072.01 \pm 6.20$ & 0.68 \\
\hline $\mathrm{AlPO}_{4}$ Berlinite & $-1,617.90 * \quad(1)$ & $-1,625.44 \pm 5.79$ & 0.46 \\
\hline & $-1,601.20 *$ & & 1.51 \\
\hline & $-1,618.00 *(14)$ & & 0.46 \\
\hline & $-1,623.30 *(14)$ & & 0.13 \\
\hline A1 $1 \mathrm{PO}_{4} \cdot 2 \mathrm{H}_{2} \mathrm{O}$ Variscite & $-2,111.40$ & $-2,103.61 \pm 5.85$ & -0.37 \\
\hline & $-2,104.20$ & & -0.03 \\
\hline $\mathrm{Al}_{4}\left(\mathrm{PO}_{4}\right)_{3}(\mathrm{OH})_{3}$ Trolleite & $-6,077.47 *$ & $-6,065.73 \pm 23.62$ & -0.19 \\
\hline $\mathrm{A}_{2} \mathrm{PO}_{4}(\mathrm{OH})_{3}$ Augelite & -2809.56 & $-2814.91 \pm 15.08$ & 0.19 \\
\hline
\end{tabular}

* Values selected to calculate $g_{i,}$ (1) Wagman et al. (1982), (2) Vieillard \& Tardy (1984), (3) Wagman (1968), (4) Latimer (1952), (5) Parker et al. (1971), (6) Naumov et al. (1971), (7) Duff (1971a), (8) Rossini et al. (1952), (9) Duff (1972), (10) Robie et al. (1979), (11) Duff (1971b), (12) Tacker \& Stormer (1989), (13) Al-Borno \& Tomson (1994), (14) Woods \& Garrels (1987).

values considered), an uncertainty level similar to the method here proposed!

Table 6 compares the calculated values of free energy at high temperature, using equation 8 , to the published experimental data of Robie et al. (1979)

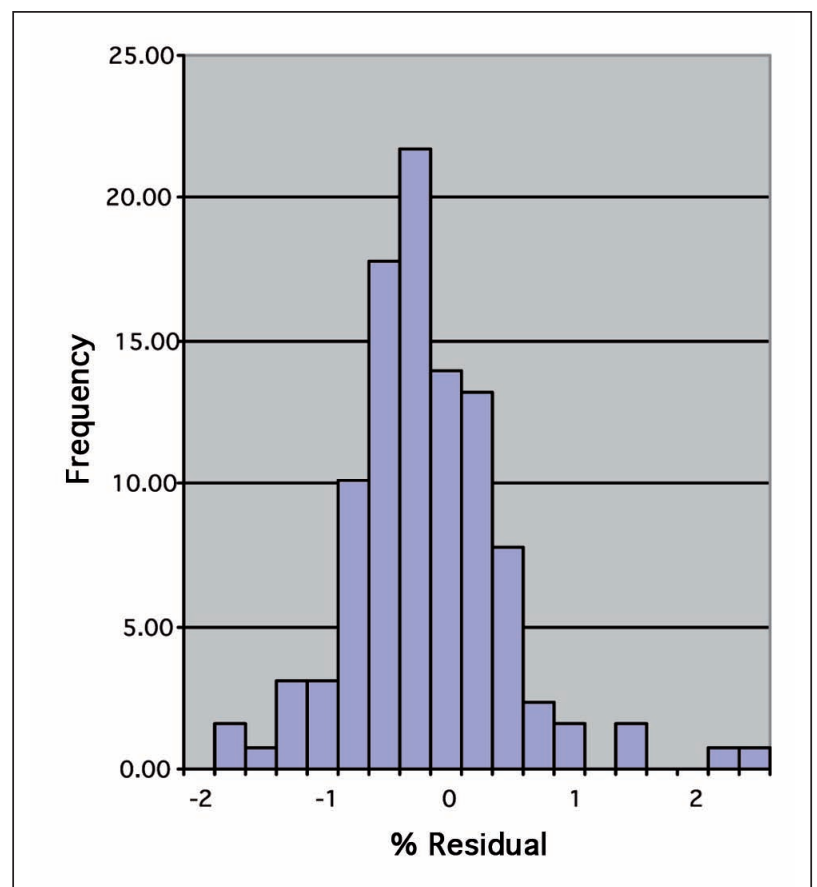

Fig. 1.-Frequency histogram of residual differences between bibliographic and calculated values of $\Delta \mathrm{G}_{\mathrm{f}}^{\circ}$ (phosphates). for berlinite, whitlockite, fluorapatite and hydroxiapatite in the temperature range of 400 to $700 \mathrm{~K}$. In all of the studied cases, the relative error is less than $0.9 \%$, with $\mathrm{R}=-0.044$ and $\sigma_{\mathrm{n}}=0.428(\mathrm{~N}=16$ values considered).

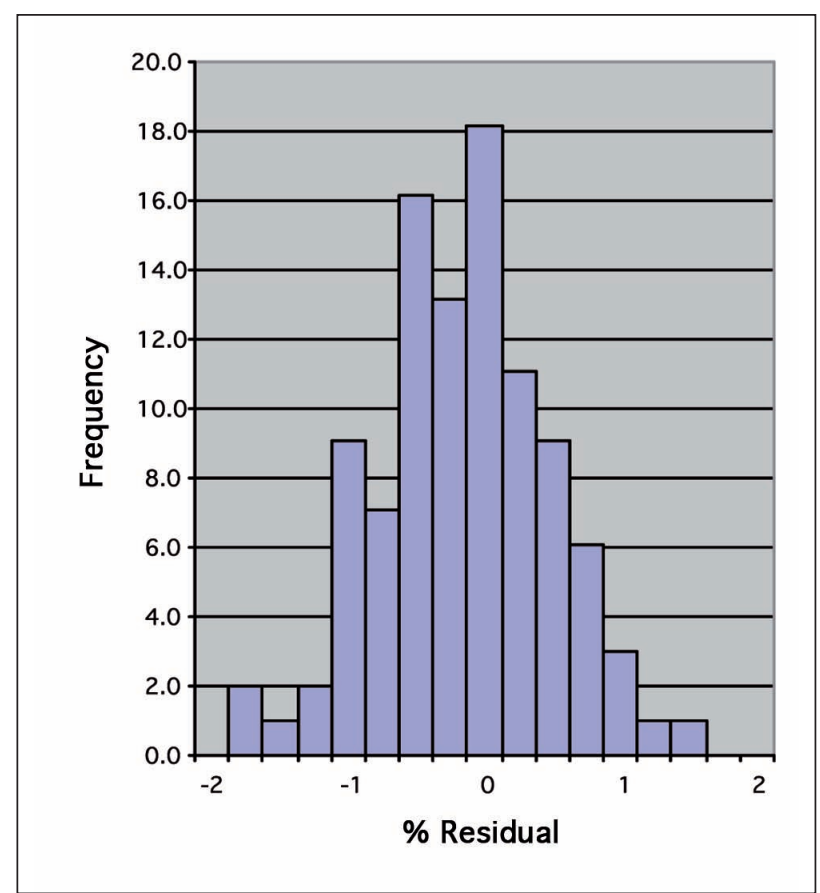

Fig. 2.-Frequency histogram of residual differences between bibliographic and calculated values of $\Delta \mathrm{H}_{\mathrm{f}}^{\circ}$ (phosphates). 
Table 5.-Comparison of $\Delta \mathbf{H}_{\mathrm{f}}{ }^{0}$ (bibliography) versus $\Delta \mathbf{H}_{\mathrm{f}}{ }^{0}$ (calculated) for phosphates and residual error $\left(\mathrm{kJ} \mathrm{mol}^{-1}\right)$

\begin{tabular}{|c|c|c|c|}
\hline \multirow{2}{*}{$\frac{\text { Crystalline species }}{\mathrm{Li}_{3} \mathrm{PO}_{4} \text { Lithiophosphatite }}$} & $\Delta \mathrm{H}_{\mathrm{f}}^{0}($ bibli. $)$ & \multirow{2}{*}{$\frac{\Delta \mathrm{H}_{\mathrm{f}}^{0}(\mathrm{Ca} 1 \mathrm{c} .)}{-2,088.32 \pm 19.79}$} & \multirow{2}{*}{$\begin{array}{r}\mathrm{R} \% \\
-0.36 \\
-0.19\end{array}$} \\
\hline & $\begin{array}{ll}-2,095.80 * & (1) \\
-2,092.20 * & (2)\end{array}$ & & \\
\hline $\mathrm{LiP}_{2} \mathrm{O}_{7}$ & $-3,356.00 *$ & $-3,360.20 \pm 26.93$ & 0.13 \\
\hline $\mathrm{LiH}_{2} \mathrm{PO}_{4}$ & $\begin{array}{ll}-1,586.60 & (2) \\
-1,573.60 & (1)\end{array}$ & $-1,579.99 \pm 7.61$ & $\begin{array}{r}-0.42 \\
0.41\end{array}$ \\
\hline $\mathrm{Na}_{3} \mathrm{PO}_{4}$ & $\begin{array}{ll}-1,917.40 & (1) \\
-1,913.60 & (2)\end{array}$ & $-1,942.32 \pm 6.25$ & $\begin{array}{l}1.30 \\
1.50\end{array}$ \\
\hline $\mathrm{Na}_{4} \mathrm{P}_{2} \mathrm{O}_{7}$ & $-3,188.00$ & $-3,165.53 \pm 9.92$ & -0.71 \\
\hline $\mathrm{Na}_{4} \mathrm{P}_{2} \mathrm{O}_{7} \cdot 10 \mathrm{H}_{2} \mathrm{O}$ & $-6,138.00 *$ & $-6,158.12 \pm 11.70$ & 0.33 \\
\hline $\mathrm{Na}_{4} \mathrm{P}_{4} \mathrm{O}_{12}$ & $-4,862.00$ & $-4,892.81 \pm 15.96$ & 0.63 \\
\hline $\mathrm{Na}_{5} \mathrm{P}_{3} \mathrm{O}_{10}$ & $-4,409.90 *$ & $-4,388.74 \pm 13.78$ & -0.48 \\
\hline $\mathrm{Na}_{5} \mathrm{P}_{3} \mathrm{O}_{10} \cdot 6 \mathrm{H}_{2} \mathrm{O}$ & $-6,194.80$ & $-6,184.29 \pm 14.26$ & -0.17 \\
\hline $\mathrm{NaH}_{2} \mathrm{PO}_{4}$ & $\begin{array}{ll}-1,536.80 & (1) \\
-1,543.90 & (2)\end{array}$ & $-1,531.33 \pm 4.32$ & $\begin{array}{l}-0.36 \\
-0.81\end{array}$ \\
\hline $\mathrm{NaH}_{2} \mathrm{PO}_{4} \cdot \mathrm{H}_{2} \mathrm{O}$ & $-1,833.00$ & $-1,830.59 \pm 4.37$ & -0.13 \\
\hline $\mathrm{NaH}_{2} \mathrm{PO}_{4} \cdot 2 \mathrm{H}_{2} \mathrm{O}$ & $-2,128.40$ & $-2,129.85 \pm 4.41$ & 0.07 \\
\hline $\mathrm{Na}_{2} \mathrm{HPO}_{4}$ & $\begin{array}{r}-1,748.10 \\
-754.80\end{array}$ & $-1,736.83 \pm 5.03$ & $\begin{array}{r}-0.64 \\
0.20\end{array}$ \\
\hline $\mathrm{Na}_{2} \mathrm{HPO}_{4} \cdot 2 \mathrm{H}_{2} \mathrm{O}$ & $-2,346.00$ & $-2,335.32 \pm 5.18$ & -0.46 \\
\hline $\mathrm{Na}_{2} \mathrm{HPO}_{4} \cdot 7 \mathrm{H}_{2} \mathrm{O}$ & $-3,821.70$ & $-3,831.57 \pm 6.64$ & 0.26 \\
\hline $\mathrm{Na}_{2} \mathrm{HPO}_{4} 12 \mathrm{H}_{2} \mathrm{O}$ & $-5,297.80 *$ & $-5,327.82 \pm 8.98$ & 0.57 \\
\hline $\mathrm{Na}_{2} \mathrm{H}_{2} \mathrm{P}_{2} \mathrm{O}_{7}$ & $-2,764.80$ & $-2,754.52 \pm 8.15$ & 0.37 \\
\hline $\mathrm{Na}_{2} \mathrm{H}_{2} \mathrm{P}_{2} \mathrm{O}_{7} \cdot 6 \mathrm{H}_{2} \mathrm{O}$ & $-4,531.00$ & $-4,550.02 \pm 8.96$ & 0.42 \\
\hline $\mathrm{Na}_{3} \mathrm{HP}_{2} \mathrm{O}_{7}$ & $-2,965.60 * \quad(1)$ & $-2,960.03 \pm 16.94$ & -0.19 \\
\hline $\mathrm{Na}_{3} \mathrm{HP}_{2} \mathrm{O}_{7} \cdot \mathrm{H}_{2} \mathrm{O}$ & $-3,276.50$ & $-3,259.29 \pm 8.90$ & -0.53 \\
\hline $\mathrm{Na}_{3} \mathrm{HP}_{2} \mathrm{O}_{7} \cdot 6 \mathrm{H}_{2} \mathrm{O}$ & $-4,740.90$ & $-4,755.52 \pm 9.63$ & 0.31 \\
\hline $\mathrm{NaNH}_{4} \mathrm{HPO}_{4} \cdot 4 \mathrm{H}_{2} \mathrm{O}$ & $-2,852.27$ & $-2,848.27 \pm 6.81$ & -0.14 \\
\hline $\mathrm{NaCaPO}_{4}$ & $-2,004.14$ & $-2,014.36 \pm 6.85$ & 0.51 \\
\hline $\mathrm{KH}_{2} \mathrm{PO}_{4}$ & $\begin{array}{ll}-1,568.33 & (1) \\
-1,570.70 & (2)\end{array}$ & $-1,550.36 \pm 4.29$ & $\begin{array}{r}-1.15 \\
1.30\end{array}$ \\
\hline $\mathrm{K}_{2} \mathrm{HPO}_{4}$ & $-1,779.90 *$ & $-1,774.89 \pm 4.93$ & 0.28 \\
\hline $\mathrm{K}_{2} \mathrm{H}_{2} \mathrm{P}_{2} \mathrm{O}_{7}$ & $-2,816.00$ & $-2,792.60 \pm 8.09$ & -0.83 \\
\hline $\mathrm{K}_{3} \mathrm{~A}_{15}\left(\mathrm{PO}_{4}\right)_{8} \mathrm{H}_{6} \cdot 18 \mathrm{H}_{2} \mathrm{O}$ Pot. Taranakite & $\begin{array}{ll}-18,908.20 & (2) \\
-18,919.20 & (1)\end{array}$ & $-18,757.23 \pm 35.91$ & $\begin{array}{l}-0.80 \\
-0.86\end{array}$ \\
\hline $\mathrm{KMgPO}_{4} \cdot 6 \mathrm{H}_{2} \mathrm{O}$ & $-3,724.30 *$ & $-3,729.29 \pm 6.92$ & 0.13 \\
\hline $\mathrm{NH}_{4} \mathrm{H}_{2} \mathrm{PO}_{4}$ & $\begin{array}{ll}-1,445.07 * & (1) \\
-1,445.10 * & (3)\end{array}$ & $-1,445.77 \pm 6.28$ & $\begin{array}{l}0.05 \\
0.05\end{array}$ \\
\hline$\left(\mathrm{NH}_{4}\right)_{2} \mathrm{HPO}_{4}$ & $-1,566.90 * \quad(1)$ & $-1,565.72 \pm 10.40$ & -0.08 \\
\hline$\left(\mathrm{NH}_{4}\right)_{3} \mathrm{PO}_{4}$ & $-1,671.90$ & $-1,685.67 \pm 15.02$ & 0.82 \\
\hline$\left(\mathrm{NH}_{4}\right)_{3} \mathrm{PO}_{4} \cdot 3 \mathrm{H}_{2} \mathrm{O}$ & $-2,555.60$ & $-2,583.42 \pm 15.13$ & 1.09 \\
\hline$\left(\mathrm{NH}_{4}\right) \mathrm{MgPO}_{4} \cdot 6 \mathrm{H}_{2} \mathrm{O}$ Struvite & $\begin{array}{ll}-3,681.90 & (1) \\
-3,681.92 & (5)\end{array}$ & $-3,624.70 \pm 8.30$ & $\begin{array}{l}-1.55 \\
-1.55\end{array}$ \\
\hline$\left(\mathrm{NH}_{4}\right)_{3} \mathrm{Mg}\left(\mathrm{PO}_{4}\right) \mathrm{H}_{6} \cdot 18 \mathrm{H}_{2} \mathrm{O}$ Amm. Taranakite & $\begin{array}{ll}-18,532.60 & (2) \\
-18,547.70 & (1)\end{array}$ & $-18,484.51 \pm 38.45$ & $\begin{array}{l}-0.26 \\
-0.34\end{array}$ \\
\hline $\mathrm{Mg}_{3}\left(\mathrm{PO}_{4}\right)_{2}$ Farringtonite & $\begin{array}{l}-3,780.70 *(1) \\
-3,780.66 *(5) \\
-3,790.30 *\end{array}$ & $-3,801.93 \pm 14.76$ & $\begin{array}{l}0.56 \\
0.56 \\
0.31\end{array}$ \\
\hline $\mathrm{Ca}_{3}\left(\mathrm{PO}_{4}\right)_{2}$ low Whitlockite & $\begin{array}{ll}-4,120.82 & (2) \\
-4,120.80 & (1) \\
-4,137.60 & (8) \\
-4,125.40 & (6) \\
-4,120.80 & (9)\end{array}$ & $-4,100.76 \pm 18.20$ & $\begin{array}{l}-0.49 \\
-0.49 \\
-0.89 \\
-0.60 \\
-0.49\end{array}$ \\
\hline $\mathrm{CaHPO}_{4}$ Monetite & $\begin{array}{l}-1,820.90 \\
-1,813.20 \\
-1,814.40\end{array}$ & $-1,808.86 \pm 6.69$ & $\begin{array}{l}-0.66 \\
-0.24 \\
-0.31\end{array}$ \\
\hline $\mathrm{CaHPO}_{4} \cdot 2 \mathrm{H}_{2} \mathrm{O}$ Brushite & $\begin{array}{ll}-2,403.60 * & (2) \\
-2,410.00 * & (8) \\
-2,402.70 * & (6)\end{array}$ & $-2,407.36 \pm 6.80$ & $\begin{array}{r}0.16 \\
-0.11 \\
0.19\end{array}$ \\
\hline
\end{tabular}

*Values selected to calculate $h_{i}$ References (1) to (14) see Table 4. 
Table 5.-Comparison of $\Delta \mathbf{H}_{\mathrm{f}}{ }^{0}$ (bibliography) versus $\Delta \mathbf{H}_{\mathrm{f}}{ }^{0}$ (calculated) for phosphates and residual error $\left(\mathrm{kJ} \mathrm{mol}^{-1}\right)$ (continuation)

\begin{tabular}{|c|c|c|c|}
\hline \multirow{2}{*}{$\frac{\text { Crystalline species }}{\mathrm{Ca}\left(\mathrm{H}_{2} \mathrm{PO}_{4}\right)_{2}}$} & $\Delta \mathrm{H}_{\mathrm{f}}^{0}($ bibli. $)$ & \multirow{2}{*}{$\frac{\Delta \mathrm{H}_{\mathrm{f}}^{0}(\mathrm{Ca} 1 \mathrm{c} .)}{-3,134.68 \pm 9.70}$} & \multirow{2}{*}{$\begin{array}{l}\mathrm{R} \% \\
0.99\end{array}$} \\
\hline & $-3,104.10$ & & \\
\hline & $-3,104.60$ & & 0.97 \\
\hline & $-3,104.70$ & & 0.97 \\
\hline & $-3,113.60$ & & 0.68 \\
\hline \multirow[t]{2}{*}{$\mathrm{Ca}\left(\mathrm{H}_{2} \mathrm{PO}_{4}\right) \cdot 2 \mathrm{H}_{2} \mathrm{O}$} & $-3,409.70$ & $-3,433.93 \pm 9.72$ & 0.71 \\
\hline & $-3,409.67$ & & 0.71 \\
\hline $\mathrm{Ca}_{2} \mathrm{P}_{2} \mathrm{O}_{7}$ & $-3,338.80$ & $-3,309.6 \pm 13.28$ & -0.88 \\
\hline \multirow[t]{8}{*}{$\mathrm{Ca}_{10}\left(\mathrm{PO}_{4}\right)_{6} \mathrm{~F}_{2}$ Fluorapatite } & $-13,744.00 *$ & $-13,679.34 \pm 60.77$ & -0.47 \\
\hline & $-13,614.08 *(12)$ & & 0.48 \\
\hline & $-13,639.72 *(10)$ & & 0.29 \\
\hline & $-13,648.20 *(12)$ & & 0.23 \\
\hline & $-13,650.70 *(12)$ & & 0.21 \\
\hline & $-13,669.96 *(12)$ & & 0.07 \\
\hline & $-13,677.48 *(12)$ & & 0.01 \\
\hline & $-13,790.46 *(12)$ & & -0.81 \\
\hline \multirow[t]{7}{*}{$\mathrm{Ca}_{10}\left(\mathrm{PO}_{4}\right)_{6}(\mathrm{OH})_{2}$ Hydroxyapatite } & $-13,338.52 *(10)$ & $-13,385.62 \pm 0.07$ & 0.35 \\
\hline & $-13,510.12 *(12)$ & & -0.92 \\
\hline & $-13,518.50 *(12)$ & & -0.98 \\
\hline & $-13,439.00 *(12)$ & & -0.40 \\
\hline & $-13,269.80 *(12)$ & & 0.87 \\
\hline & $-13,280.68 *(12)$ & & 0.79 \\
\hline & $-13,415.14 *(12)$ & & -0.22 \\
\hline \multirow[t]{3}{*}{$\mathrm{Ca}_{10}\left(\mathrm{PO}_{4}\right)_{6} \mathrm{C}_{12}$ Chiorapatite } & $-13,271.64 *(12)$ & $-13,184.10 \pm 67.56$ & -0.66 \\
\hline & $-13,272.00 *$ & & -0.66 \\
\hline & $-13,096.54 *(12)$ & & 0.67 \\
\hline $\mathrm{Fe}_{3}\left(\mathrm{PO}_{4}\right)_{2} \cdot 8 \mathrm{H}_{2} \mathrm{O}$ Vivianite & $-5,078.09 *(13)$ & $-5,078.09 \pm 10.21$ & 0.00 \\
\hline \multirow[t]{2}{*}{$\mathrm{Zn}_{3}\left(\mathrm{PO}_{4}\right)_{2}$} & $-2,896.60$ & $-2,902.65 \pm 14.16$ & 0.21 \\
\hline & $-2,892.40$ & & 0.35 \\
\hline $\mathrm{Zn}_{2} \mathrm{P}_{2} \mathrm{O}_{7}$ & $2,510.40 *$ & $-2,510.86 \pm 10.87$ & 0.02 \\
\hline $\mathrm{Zn}_{3}\left(\mathrm{PO}_{4}\right) \cdot 2 \mathrm{H}_{2} \mathrm{O}$ & $-3,211.70$ & $-3,201.91 \pm 14.17$ & -0.31 \\
\hline $\mathrm{Zn}_{3}\left(\mathrm{PO}_{4}\right)_{2} \cdot 2 \mathrm{H}_{2} \mathrm{O}$ & $-3,516.30$ & $-3,501.17 \pm 14.21$ & -0.43 \\
\hline $\mathrm{Zn}_{3}\left(\mathrm{PO}_{4}\right)_{2} \cdot 4 \mathrm{H}_{2} \mathrm{O}$ Hopeite & $-4,091.50 * \quad(2)$ & $-4,099.68 \pm 14.27$ & 0.20 \\
\hline $\mathrm{Cu}_{3}\left(\mathrm{PO}_{4}\right)_{2}$ & $-2,231.40 *(14)$ & $-2,242.08 \pm 12.55$ & 0.48 \\
\hline $\mathrm{Cu}\left(\mathrm{H}_{2} \mathrm{PO}_{4}\right)_{2}$ & $-2,526.70 *$ & $-2,515.12 \pm 8.65$ & -0.46 \\
\hline $\mathrm{Cu}_{3}\left(\mathrm{PO}_{4}\right)_{2} \cdot 2 \mathrm{H}_{2} \mathrm{O}$ & $-2,842.20$ & $-2,840.58 \pm 12.61$ & -0.06 \\
\hline $\mathrm{Cu}_{3}\left(\mathrm{PO}_{4}\right)_{2} \cdot 3 \mathrm{H}_{2} \mathrm{O}$ & $-3,139.70$ & $-3,139.83 \pm 12.68$ & 0.00 \\
\hline $\mathrm{Pb}_{3}\left(\mathrm{PO}_{4}\right)_{2}$ & $-2,595.30 *(14)$ & $-2,595.30 \pm 3.22$ & 0.00 \\
\hline \multirow[t]{2}{*}{$\mathrm{AlPO}_{4}$ Berlinite } & $-1,733.80 * \quad(1)$ & $-1,752.14 \pm 4.93$ & 1.06 \\
\hline & $-1,733.85 * \quad(2)$ & & 1.06 \\
\hline $\mathrm{A} \mathrm{PO}_{4} \cdot 2 \mathrm{H}_{2} \mathrm{O}$ Variscite & $-2,353.30$ & $-2,350.64 \pm 5.09$ & -0.11 \\
\hline $\mathrm{Al}_{4}\left(\mathrm{PO}_{4}\right)_{3}(\mathrm{OH})_{3}$ Trolleite & $-6,567.67 * \quad(2)$ & $-6,583.19 \pm 19.51$ & 0.24 \\
\hline
\end{tabular}

* Values selected to calculate $h_{i}$ References (1) to (14) see Table 4.

Table 6.-Experimental and calculated data of $\Delta \mathrm{G}_{\mathrm{f}, \mathrm{T}}\left(\mathrm{kJ} \mathrm{mo1}^{-1}\right)$

\begin{tabular}{lccccccccr}
\hline & \multicolumn{2}{c}{$400 \mathrm{~K}$} & \multicolumn{2}{c}{$500 \mathrm{~K}$} & \multicolumn{2}{c}{$600 \mathrm{~K}$} & & \multicolumn{2}{c}{$700 \mathrm{~K}$} \\
\cline { 2 - 9 } & $(1)$ & $(2)$ & $(1)$ & $(2)$ & $(1)$ & $(2)$ & $(1)$ & $(2)$ \\
\hline $\mathrm{AlPO}_{4}$ Berlinite & $-1,568.01$ & $-1,583.88$ & $-1,530.86$ & $-1,541.38$ & $-1,493.88$ & $-1,498.88$ & $-1,457.13$ & $-1,456.38$ \\
$\mathrm{Ca}_{3}\left(\mathrm{PO}_{4}\right)_{2}$ Whitlockite & $-3,783.85$ & $-3,781.27$ & $-3,708.57$ & $-3,700.27$ & $-3,633.63$ & $-3,619.27$ & $-3,559.16$ & $-3,538.27$ \\
$\mathrm{Ca}_{5}\left(\mathrm{PO}_{4}\right)_{3}$ F Fluorapatite & $-6,331.51$ & $-6,318.86$ & $-6,210.19$ & $-6,188.66$ & $-6,089.65$ & $-6,058.46$ & $-5,969.91$ & $-5,928.26$ \\
$\mathrm{Ca}_{5}\left(\mathrm{PO}_{4}\right)_{3}$ OH Hydroxyapatite & $-6,155.33$ & $-6,159.91$ & $-6,027.76$ & $-6,027.91$ & $-5,901.11$ & $-5,895.91$ & $-5,775.32$ & $-5,763.91$ \\
\hline
\end{tabular}

(1) Robie et al. (1979); (2) Calculated using values of Table 3. 


\section{Conclusions}

The method proposed by Chermak \& Rimstidt (1989) for calculation of free energy and enthalpy of silicates, by summing the contributions of polihedral units, is applicable to the estimation of $\Delta \mathrm{G}_{\mathrm{f}}^{\circ}$ and $\Delta \mathrm{H}_{\mathrm{f}}^{\circ}$ of phosphate minerals. This method allowed us to calculate the thermodynamic properties of phosphates with more accuracy than the methods published until now, with the following aditional advantages: $a$ ) use of a simple easy computed mathematical procedure, and $b$ ) the ability to obtain a wider field of application among the phosphates.

The calculation of free energy at high temperature proposed by Chermak \& Rimstidt (1990) for silicates is also applicable to phosphates, giving values very close to the experimental data.

\section{ACKNOWLEDGEMENTS}

This work was funded by project CGL-2005-C02-02 from the Spanish Ministerio de Educacion y Ciencia. The author is in debt to Prof. A. Suárez Granero, Dpto. Análisis Matemático, Facultad Ciencias Matemáticas U.C.M., Madrid, for stimulating discussion of this paper.

\section{References}

Al-Borno, A. \& Tomson, M.B. (1994). The temperature dependence of the solubility product constant of vivianite. Geochimica et Cosmochimica Acta, 58: 5373-5378. doi:10.1016/0016-7037(94)90236-4

Chen, C.H. (1975). A method for estimation of standard free energies of formation of silicate minerals at 298.15 degrees K. American Journal of Science, 275: 801-807.

Chermak, J.A. \& Rimstidt, J.D. (1989). Estimating the thermodynamic properties $\left(\Delta \mathrm{G}_{\mathrm{f}}^{\circ}\right.$ and $\left.\Delta \mathrm{H}_{\mathrm{f}}^{\circ}\right)$ of silicate minerals at $298 \mathrm{~K}$ from the sum of polyhedral contributions. American Mineralogist, 74: 1023-1031.

Chermak, J.A. \& Rimstidt, J.D. (1990). Estimating the free energy of formation of silicate minerals at high temperatures from the sum of polyhedral contributions. American Mineralogist, 75: 1376-1380.

Duff, E.J. (1971a). Orthophosphates VIII. The Transformation of newberyite into bobierrite in aqueous alkaline solutions. Journal of the Chemical Society A: 2736-2740. doi:10.1039/j19710002736

Duff, E.J. (1971b). Orthophosphates III. The hydrolysis of secondary calcium orthophosphates. Journal of the Chemical Society A: 917-921. doi:10.1039/j19710000917

Duff, E.J. (1971c). Orthophosphates. Part IV. The stability relationships of orthophosphates within the systems $\mathrm{CaO}-\mathrm{P}_{2} \mathrm{O}_{5}-\mathrm{H}_{2} \mathrm{O}$ and $\mathrm{CaF}_{2}-\mathrm{CaO}-\mathrm{P}_{2} \mathrm{O}_{5}$ under aqueous conditions. Journal of the Chemical Society A: 921926. doi:10.1039/j19710000921

Duff, E.J. (1972). Orthophosphates VII. Thermodynamical considerations concerning the stability of oxiapatite $\mathrm{Ca}_{10} \mathrm{O}\left(\mathrm{PO}_{4}\right)_{6}$ in aqueous media. Journal of Inorganic and Nuclear Chemistry 34: 853-857. doi:10.1016/0022-1902(72)80059-9

Grimwall, G. (2001). Dependence of thermodynamic properties on atomic masses and bonding in solids. In: Solid Solutions in Silicate and Oxide Systems (Geiger C.A., ed.), EMU Notes in Mineralogy, 3: 13-26.

Hazen, A.M. (1985). Comparative crystal chemistry and the polyhedral approach. In: Microscopic to macroscopic: Atomic environment to mineral thermodynamic (Kieffer, S.W. \& Navrotsky, A. eds.), Mineralogical Society of America. Reviews in Mineralogy, 14: 317-345.

Holland, T.J.B. (1989) Dependence of entropy on volume for silicate and oxide minerals: A review and predictive model. American Mineralogist, 74: 5-13.

Karpov, I.K. \& Kashik, S.A. (1968). Computer calculation of standard isobaric isothermal potentials of silicates by multiple regression from a crystallochemical classification. Geokhimiya, 7: 806-814.

La Iglesia, A. \& Aznar, A. (1986). A method of estimating the Gibbs energies of formation of zeolites. Zeolites, 6: 26-29. doi:10.1016/0144-2449(86)90007-2

La Iglesia, A. \& Aznar, A. (1990). Estimation des energies libres de Gibbs de formation de zéolithes, feldspaths et feldspathoides. Journal de Chimie Physique, 87: 1681-1689.

La Iglesia, A. \& Felix, J.F. (1994). Estimation of thermodynamic properties of mineral carbonates at high and low temperatures from the sum of polyhedral contributions. Geochimica et Cosmochimica Acta, 58: 39833991. doi:10.1016/0016-7037(94)90261-5

Latimer, W.M. (1952). Oxidation Potential, Second edition, Prentice-Hall, New York, 392 pp.

Naumov, G.B., Ryzenko, B. \& Khodakovsky, I.L. (1971). Handbook of thermochemical data. Moscow, Atomizdat, 239 pp.

Nriagu, J.D. (1975). Thermochemical approximations for clay minerals. American Mineralogist, 60: 834-839.

Nriagu, J.D. (1976). Phosphate-clay mineral relation in soil and sediments. Canadian Journal of Earth Sciences, 13: 717-736. doi:10.1139/e76-077

Parker, V.B., Wagrnan, D.D. \& Evans, W.H. (1971). Selected Values of Chemical Thermodynamic Properties. Nat. Bur. Standard. Tech. Note 270-6, 119 pp.

Robie, R.A., Hemingway, B. S. \& Fischer, J.R. (1979). Thermodynamic Properties of Minerals and Related Substances at $298.15 \mathrm{~K}$ and 1 Bar (10 $10^{5}$ Pascals) Pressure and at Higher Temperatures. Geological Survey Bulletin, 1452, 456 pp.

Robinson, G.R. Jr. \& Haas, J.L. Jr. (1983). Heat capacity, relative enthalpy, and calorimetric entropy of silicate minerals: an empirical method of prediction. American Mineralogist, 68: 541-553.

Rossini, F.D., Wagman, D.D., Evans, W.H., Levine, S. \& Jaffe, I. (1952). Selected Values of Chemical Ther- 
modynamic Properties. Nat. Bur. Standard. Circ. 500 U.S. Dept. Commerce.

Slaughter, M. (1966). Chemical binding in silicate minerals. I. Model for determining crystal-chemical properties. Geochim. et Cosmochim. Acta, 30: 299-313. doi:10.1016/0016-7037(66)90004-4

Sposito, G. (1986). The Polymer Model of Thermochemical Clay Minerals Stability. Clays and Clay Minerals, 34: 198-203. doi:10.1346/CCMN.1986.0340210

Tacker, R.C. \& Stormer, J.C. Jr. (1989). A thermodynamic model for apatite solid solutions, applicable to high-temperature geologic problems. American Mineralogist, 74: 877-888.

Tardy, Y. \& Garrels, R.M. (1974). A method of estimating the Gibbs energies of formation of layer silicates. Geochimica et Cosmochimica Acta, 38: 1101-1116. doi:10.1016/0016-7037(74)90007-6

Tardy, Y. \& Garrels, R.M. (1976). Prediction of Gibbs energies of formation: I. Relationship among Gibbs energies of formation of hydroxides, oxides and aqueous ions. Geochim. et Cosmochim. Acta, 40: 10511056. doi:10.1016/0016-7037(76)90046-6

Tardy, Y. \& Garrels, R.M. (1977). Prediction of Gibbs energies of formation of compounds from the elements: II. Monovalent and divalent metal silicates. Geochim. et Cosmochim. Acta, 41: 87-92. doi:10.1016/0016-7037(77)90189-2

Tardy, Y. \& Gartner, L. (1977). Relationships among Gibbs energies of formation of sulfates, nitrates, carbonates, oxides and aqueous ions. Contributions to Mineralogy and Petrology, 63: 89-102. doi:10.1007/BF00371678
Tardy, Y. \& Vieillard, P. (1977). Relationships among Gibbs Free Energies and Enthalpies of Formation of Phosphates, Oxides and Aqueous Ions. Contributions to Mineralogy and Petrology, 63: 75-88. doi:10.1007/BF00371677

Vieillard, P. \& Tardy, Y. (1984). Thermochemical Properties of Phosphates. In: Phosphate Minerals (Nriagu, J.O. \& Moore, P.B., eds.), Springer-Verlag, Berlin, $442 \mathrm{pp}$.

Wagman, D.D., Evans, W.H., Parker, V.B., Halow, I., Bailey, S.M. \& Schumm, R.H. (1968). Selected Values of Chemical Thermodynamic Properties, National Bureau of Standards, Technical Note 270-4, 152 pp.

Wagman, D.D., Evans, W.H., Parker, v. B., Schumm, R.H., Halow, I., Bailey, S.M., Churney, I.C.L. \& Nuttall, R.L. (1982). The NBS tables of chemical thermodynamic properties: Selected values for inorganic and $\mathrm{C}_{1}$ and $\mathrm{C}_{2}$ organic substances in SI units. Journal of Physical and Chemical Reference Data, 11, Supplement 2, 392 pp.

Wilcox, D.E. \& Bromley, R.A. (1963). Method for Estimating the Heat of Formation and Free Energy of Formation of Inorganic Compounds. Ind. Eng. Chem., 55: 32-39. doi:10.1021/ie50643a006

Woods, T.L. \& Garrels, R.M. (1987). Thermodynamic values at low temperature for natural inorganic materials. An uncritical summary. Oxford University Press, $242 \mathrm{pp}$.

Recibido el 05 de febrero de 2009 Aceptado el 23 de junio de 2009 Publicado online el 29 de diciembre de 2009 